\title{
Future GNSS: Improved Signals and Constellations
}

\author{
Guillermo Martínez Morán ${ }^{1}$ \\ ${ }^{1}$ Airbus Defense \& Space. Paseo John Lennon s/n - 28096 Getafe (Madrid - Spain) \\ Guillermo.M.Martinez@military.airbus.com
}

\begin{abstract}
:
December 31, 2020 in the GNSS (Global Navigation Satellite Systems) world. The U.S. Government has just switched off the military encrypted signal embedded in the L2 and L1 frequency of the GPS system. All the high precision receivers based on L2 codeless and semi-codeless acquisition have become obsolete. Luckily, new improved GPS signals are available both in L2 and the new L5 frequency. In addition, new GNSS systems have become operative. The European Union has deployed GALILEO, CHINA Compass and Russia has upgraded GLONASS with CDMA signals.

This paper briefly explains the new scenario in satellite positioning systems that will appear in the following years. New modernized signals and their expected improvements and performance are covered. Besides, new systems features and constellations are also explained.
\end{abstract}

Key words: GNSS, L2C, L5, L1C, MBOC, AltBOC.

\section{Introduction}

Since the launching of first GPS and GLONASS satellites, 30 years ago, scientist and engineers all over the world have exploit the possibilities of the system to develop many applications from personal LBS (Localization Based Services) to accurate geodesy studies. In some way, it can be assured that the current GNSS state-of-the-art has almost reached the top performance that present systems can offer.

Therefore, in order to continue improving GNSS possibilities, a modernization of the systems becomes imperative. Right now, we are living the deployment phase of the new systems, which end date can be fixed in December $31^{\text {st }}$ 2020. That is the date when legacy military $P(Y)$ signal will be shutdown, making the current flight test high accurate receivers complete obsolete.

Fortunately, by then, a lot of new GNSS satellites broadcasting improved signals will take over the duty of accurate positioning with unknown performance. We are, then, facing an exciting epoch for engineers similar to the 80's when the 3D, real time positioning appears.

\section{The future is taking shape above your head!}

This paper summarizes the main improvements expected taking into account several aspects.

Firstly, the impact of the new constellations and benefits from the increased number of satellites are analyzed.
Secondly, main characteristics and expected improvements of the new signals (L2C, L5, L1C) are addressed.

Thirdly, the advantages of the new interoperable modulations MBOC (Multiplexed Binary Offset Carrier) and AltBOC (Alternative $B O C$ ) are briefly explained.

Fourthly, the typical flight test GNSS scenario is described and the main advantages, that new systems may bring are analyzed.

Finally, it is discussed how all this evolution will impact in the receivers market in terms of features and prices.

The final Appendix summarizes the way the new modulations are formed.

\section{Satellite Constellations}

Legacy situation

Before year 2010 the only available positioning system was GPS, as GLONASS did not have enough infrastructure to provide a quality service after the USSR collapse.

\section{Current situation}

Nowadays, GPS and GLONASS are both fulloperative and there are many receivers taking advantage of using both. The main benefit is not better accuracy, but an improvement in solution availability in challenge scenarios (urban canyons, forests, etc.) 
As these scenarios do not exist in the flight test context, GPS is the only constellation normally used for flight test applications.

\section{Future situation}

Current deployment plans for the main four GNSS constellations allow an estimation of between 90-120 positioning satellites in year 2020 (see Tab. 1). Such important number of satellites will improve for sure positioning possibilities in forest and cities. In addition to the signal availability, PDOP (Dilution of precision) will be the other improved parameter.

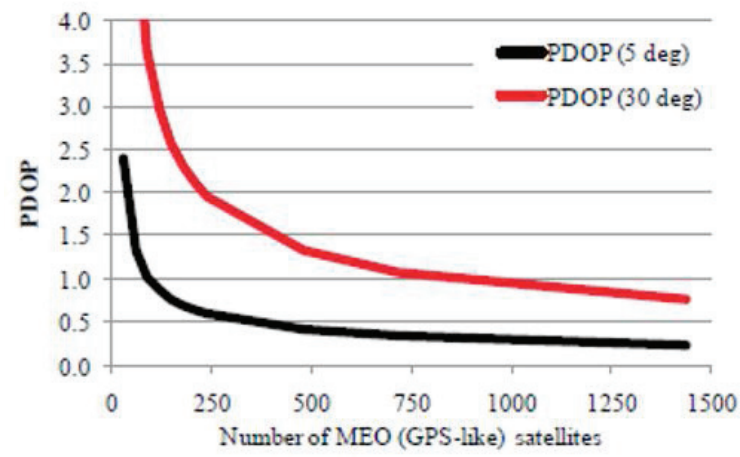

Fig. 1.- PDOP asymptotic curve

The PDOP is a constellation geometry dependant factor that multiplies the errors performed when measuring distance between receiver and satellites. Therefore, the lower it is the better for the positioning accuracy.

Experimentally, it has been shown that PDOP values below 4 are good enough, so in an open sky environment it has never been a problem, except in very extreme latitudes. This is the reason why in stabilized flight test applications PDOP has never been an issue.

In general, a lot of satellites available will allow receivers to choose those providing a better geometry, thus improving the accuracy.

Fig. 2, Fig. 3, Fig. 4 show the worldwide mean PDOP value taking into account different constellations. They illustrate the improvement in the PDOP as more satellites are added. However, as it can be appreciate in Fig. 1, there is an asymptotic dependency, from which a reasonable limit for satellites addition can be established.

The big question is: having more satellites will benefit flight test applications? You can find the answer in the last section.

\section{Positioning Signals}

Legacy situation

Up to mid 2000s the only open positioning signal in the GPS constellation was C/A in L1.
Nevertheless, clever codeless and semicodeless techniques allowed the use of military encrypted signals in L2 for high precision applications, not without some drawbacks as losing L2 signal power in the receiver [1] (3.1).

In addition, the legacy GPS signal structure does not have a separate pilot signal. This fact limits the capability to detect the signals coming from satellites, as the integration time is limited to $20 \mathrm{~ms}$ (duration time of one bit from the navigation message) [2] (Chap.7).

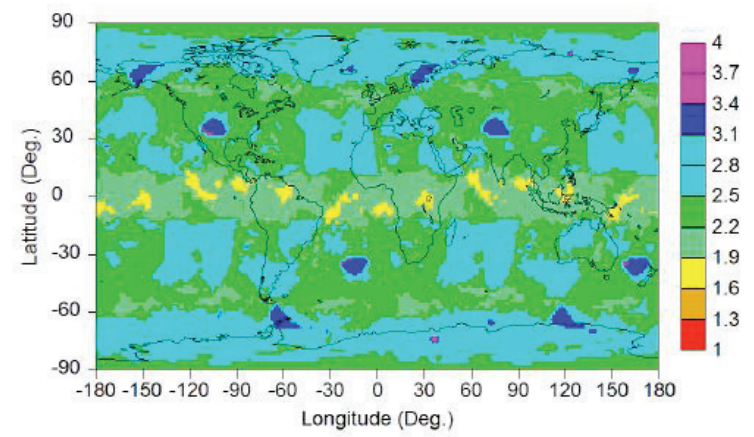

Fig. 2.- Current GPS PDOP model ( $0^{\circ}$ Mask)

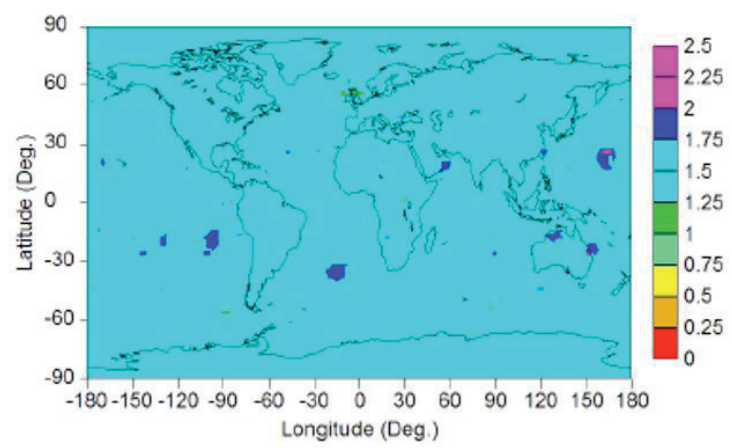

Fig. 3.- Current GPS+GLONASS PDOP model $10^{\circ}$ Mask)

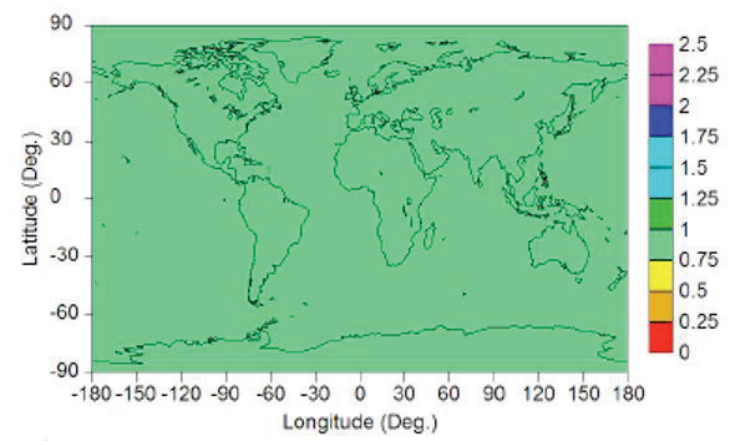

Fig. 4.- Future GPS + GLONASS + COMPASS + GALILEO PDOP model (0 Mask)

\section{Current situation}

Currently, GPS constellation is under modernization and signals solving some of the drawbacks are being added. However, as this is a slow process, not all the satellites are equipped with modernized signals. Tab. 1 
shows signals and satellites available now and in 2020 .

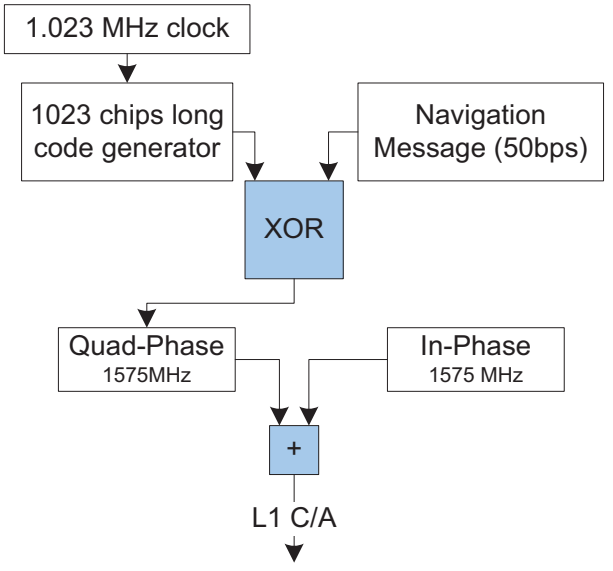

Fig. 5.- L1 C/A Signal Structure

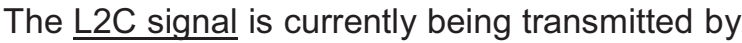
11 satellites and it is not necessary to have 4 L2C satellites in view to take advantage of it, as each satellite in view improved our position in terms of reliability and precision. For the first time, regular users can access an open signal in a second frequency, allowing a better correction of the ionosphere delay error [1](1.4).

Besides, this signal provides a pilot signal without navigation message inside, which allows a better detection of the signal, which is very useful in harsh environments. Although signal power is not too strong, reception level is higher than legacy L2 as codeless techniques are not necessary.

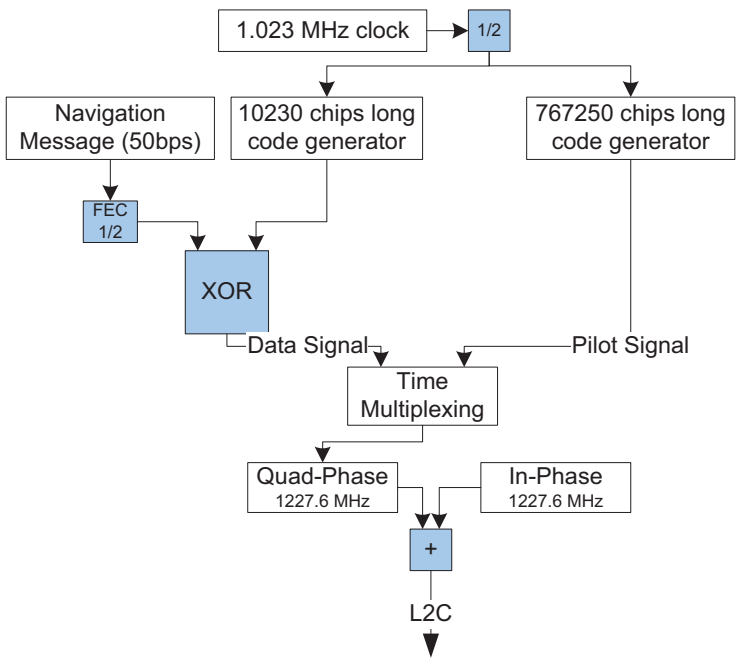

Fig. 6.- L2C Signal Structure

Another improvement to make the signal more reliable is the possibility to code the navigation message with a FEC 1/2 (Forward Correction Error Code), allowing data recovery in case of any data failure during the reception.
The code rate is not improve respect to $L 1 \mathrm{C} / \mathrm{A}$ as it remains in $1.023 \mathrm{MHz}$ (BPSK(1) where $1=1 * 1023)$. Therefore, pseudorange resolution to the satellite is still 293 meters.

\section{Future situation}

The $\mathrm{L} 5 \mathrm{C}$ signal is being transmitted by 4 satellites at the present time. As it is mandatory to have 4 satellites in view to use this signal, it is more a future signal than a present one.

L5C has been design to meet Safety-of-Life requirements. For this reason, the transmission frequency is inside the ARNSS (Aeronautical Radio Navigation-Satellite Service) band, which is more protected against interferences.

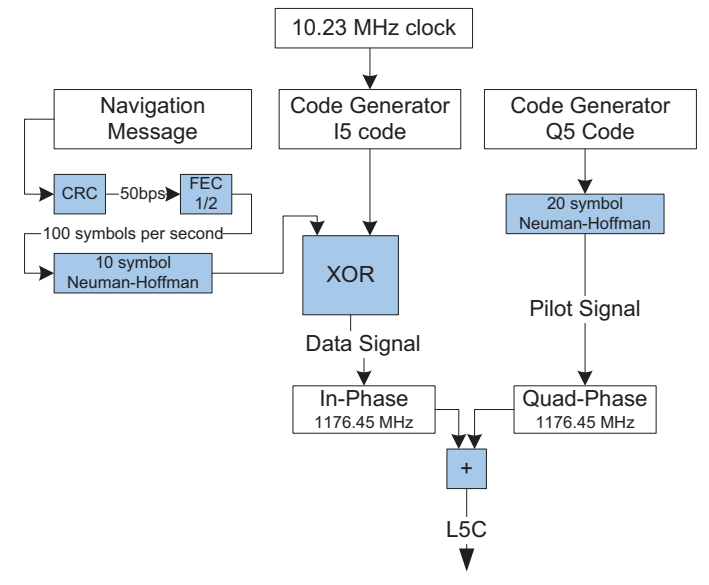

Fig. 7.- L5C Signal Structure

Minimum signal power level is about $7 \mathrm{~dB}$ more than $\mathrm{L} 2 \mathrm{C}$ and similar to $\mathrm{L} 1 \mathrm{C} / \mathrm{A}$, so reception will be easier. In addition, there is a pilot signal and a data signal as in $\mathrm{L} 2 \mathrm{C}$, which is expected to boost signal acquisition and reliability.

Data rate for the spreading code is 10 times higher than in the other signals (10.23 MHz), so pseudorange resolution to the satellite improves to 29 meters. Moreover, as the signal has more bandwidth, it is more robust against multipath, as expose in next section.

Once L2C and L5 are fully operational, codeless and semi-codeless techniques will be overcome by them and military $P(Y)$ signal at L2 frequency will be discontinued.

The $4^{\text {th }}$ open service signal, L1C signal, available from 2016, according to present schedule, will coexists with the rest of the signals including L1 C/A. This signal has been designed with same code rates as $\mathrm{L} 1 \mathrm{C} / \mathrm{A}$, but with separated pilot and data signals and with a MBOC modulation in order to be compatible with GALILEO system. Next section explains the advantages of these new modulations. 
In addition, the European constellation GALILEO will be operative providing open services in both E1 and E5 frequencies (same as L1 and L5).

E1 open service is very similar to the $\mathrm{L} 1 \mathrm{C}$ with $1.023 \mathrm{MHz}$ spreading code rate, separated data and pilot channels and similar minimum power to the $\mathrm{L} 1 \mathrm{C}$. As in the case of $\mathrm{L} 1 \mathrm{C}$, modulation is also based in MBOC.

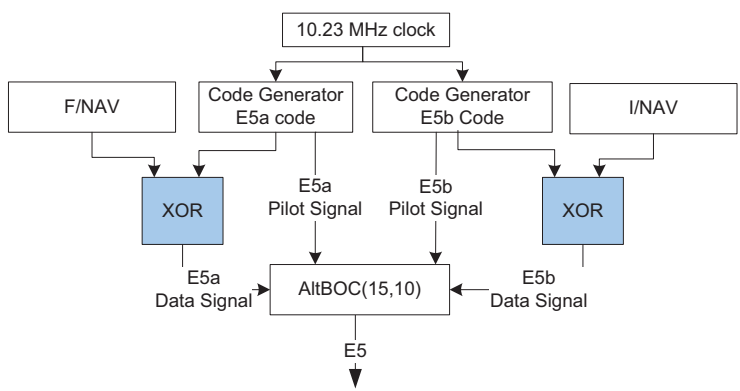

Fig. 8.- E5 Signal Schema

E5 signal is divided in two different services E5a and E5b. Both services use same signal schema, but the navigation message transmitted are different.
E5a transmit F/NAV (Freely Accessible Navigation Message), designed to provide positioning services [3].

E5b transmit I/NAV (Integrity Navigation Message) providing time-alarms and integrity information to be used in Safety-of-Life applications [3].

\section{Modulations}

New improved GNSS modulations look for the improvement of two important characteristics: better multipath performance and easier signal tracking.

A better multipath performance means a reduction in the number of reflected signals affecting the straight path signal. Typically, reducing the distance from where a reflected signal can affect. Fig. 9 shows the distance envelope for different modulations.

It can be seen how BPSK(1)[1.023MHz] is affected by $1 \mathrm{~ms}$ delayed signals (which is an obstacle at $300 \mathrm{~m}$ ), but for BPSK(10)[10.23MHz] the envelope is reduced to $75 \mathrm{~ms}$ (obstacle at $23 \mathrm{~m}$ ). Therefore, the more bandwidth, the better multipath performance.

\begin{tabular}{|c|c|c|c|c|c|c|}
\hline & & & & \multicolumn{2}{|c|}{ Sats \# } & Min.Signal \\
\hline & Signal & Modulation & Frequency $(\mathrm{MHz})$ & Present & 2020 & Receiver \\
\hline \multirow{5}{*}{$\begin{array}{l}\text { GPS } \\
\text { (USA) }\end{array}$} & L1 C/A & BPSK(1) & 1575.42 & 31 & 31 & $-158.5 \mathrm{dBW}$ \\
\hline & L1C & $\operatorname{MBOC}(6,1,1 / 11)$ & 1575.42 & 0 & ¿? & $-157.0 \mathrm{dBW}$ \\
\hline & L2C & $\operatorname{BPSK}(1)$ & 1227.6 & 11 & 31 & $-164.5 \mathrm{dBW}$ \\
\hline & L5C & QPSK & 1176.45 & 4 & 24 & $-157.9 \mathrm{dBW}$ \\
\hline & & & TOTAL & 31 & 31 & \\
\hline & L1OF & BPSK(FDMA) & $1598.06-1604.40$ & & & $-161.0 \mathrm{dBW}$ \\
\hline $\begin{array}{l}\text { GLONASS } \\
\text { (Russia) }\end{array}$ & L2OF & BPSK(FDMA) & $1242.94-1248.63$ & 24 & 30 & $-167.0 \mathrm{dBW}$ \\
\hline & L3OC & BPSK(10) & $1202.025 / 1207.14$ & & & ¿? \\
\hline & $\mathrm{B} 1(\mathrm{I})$ & QPSK & 1561.10 & & & ¿? \\
\hline COMPASS & $\begin{array}{l}\text { B1- } \\
\text { BOC }\end{array}$ & $\operatorname{MBOC}(6,1,1 / 11)$ & 1575.42 & & & ¿? \\
\hline (China) & $\mathrm{B} 2(\mathrm{I})$ & QPSK & 1207.14 & 14 & 30 & ¿? \\
\hline & $\begin{array}{l}\text { B2- } \\
\text { BOC }\end{array}$ & AltBOC $(10,5)$ & 1207.14 & & & ¿? \\
\hline & E5a & AltBOC $(15,10)$ & 1176.45 & & & $-155.0 \mathrm{dBW}$ \\
\hline $\begin{array}{l}\text { GALILEU } \\
\text { (UE) }\end{array}$ & E5b & & 1207.14 & 4 & $\begin{array}{l}10 \\
\text { (or }\end{array}$ & $-155.0 \mathrm{dBW}$ \\
\hline & E1 & $\operatorname{MBOC}(6,1,1 / 11)$ & 1575.42 & & & $-157.0 \mathrm{dBW}$ \\
\hline
\end{tabular}

All numbers in modulations refers to multiplication factor respect fundamental GNSS frequency $1.023 \mathrm{MHz}$

Tab. 1: GNSS Open Services Summary 
Nevertheless, more bandwidth also increments receiver complexity and cost. By designing a clever modulation is possible to achive equal performance to the $\operatorname{BPSK}(10)$, but using less bandwidth. MBOC spectrum make it and TMBOC (Time MBOC) and CBOC (Complex $B O X)$ are two different implementations in the time domain to get MBOC spectrum.

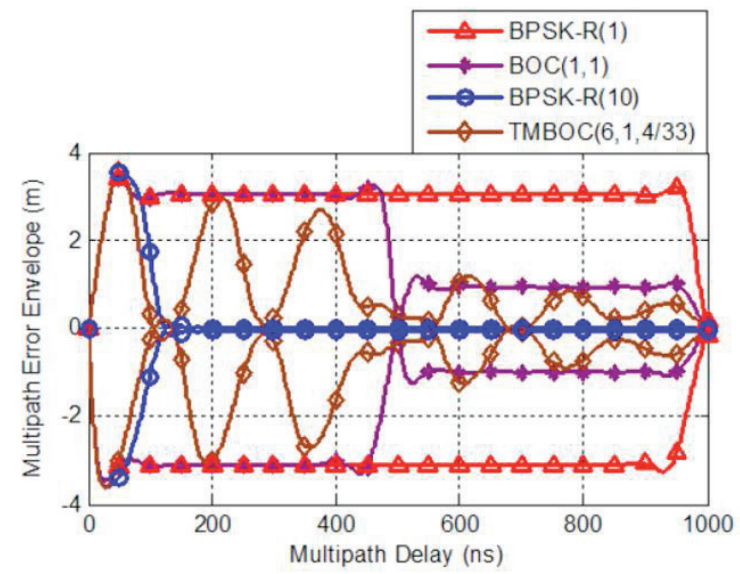

Fig. 9.- Multipath performance of different modulations

An easier signal tracking reduce the probability for the receiver to switch tracking to a reflected signal. The most important signal parameter to achieve this characteristic is the slope of the correlation peak for the modulation. The steeper the slope is the easier the tracking [4].

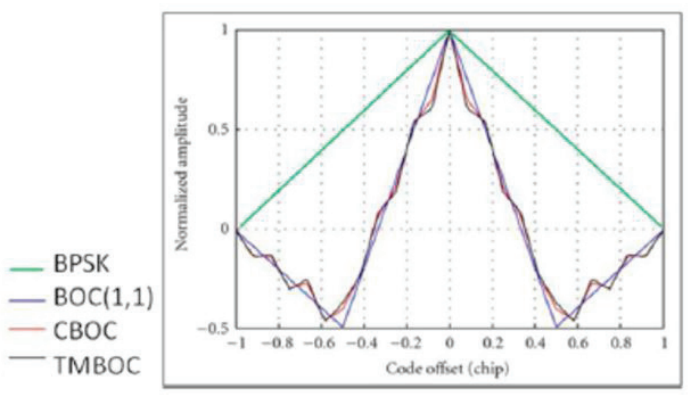

Fig. 10.- Correlation peak for several modulations.

Fig. 10 shows the big different in the slope between BPSK and the two MBOC type modulations.

\section{Impact in Flight Test applications}

Flight test applications are always in a clear sky environment, so typical GNSS signal obstruction does not happen here.

Nevertheless, it is quite normal to lose precise trajectography when performing banked turns or stall maneuvers, due to the obscuration of some satellites.

This situation will be highly alleviated when more satellite options are available, as it is quite probable to have at least 4 satellites in view even when seeing only a part of the sky. It is true that the PDOP in such situation will be quite high, but at least a solution will be available.

As stated before, new L5 and E5 signals will be allocated in the ARNSS part of the spectrum improving the protection against interference.

However, this may be an issue in our context, as placement for the GPS antenna becomes critical in order to avoid interferences coming from other aircraft systems, improving installation complexity.

On the other hand, multipath in an aircraft environment is high. Placing an antenna just on a big metallic surface, surrounded by other metallic surfaces impacts negatively in high precision applications. New improved signals will provide a better performance against these enemies.

First, only signals coming from 23 meters at most will affect performance, reducing the number of possible interferences.

Besides, easier tracking signals will decrease the probability to switch to a reflected signal once the original signal is being tracked.

\section{Market foreseen}

The changes in GNSS described in this paper, and many others outside them, will heavily impact the receivers market in the next few years, especially high accuracy market receivers.

In author's opinion, there are two main factors to be taken into account: the high number of signals and systems available and the new open accessible signals.

It is not probable to find many receivers capable of tuning all the possible existing constellations, as this would be very expensive to design and manufacture. The most probable scenario will be different series of receivers being able to track two different constellations. Furthermore, when having three constellations do not improve significantly PDOP values.

For flight test applications, I would bet for a solution with receivers acquiring both GPS and GALILEO constellations.

Among all the possible signals provided, I would bet for the use of L1C and L5 in GPS and $\mathrm{E} 1$ and $\mathrm{E} 5 \mathrm{a}$ in GALILEO. All of them are transmitted in the same frequency range, simplifying the radio part of the receiver. Moreover, as they are the most apart signals in the frequency spectrum, they are the ones that 
can achieve the best correction for the ionosphere delay. Taking this into account, it is quite probable that the manufacturers select this set of data for being the best cost/performance figure.

On the other hand, it is important to notice that all these signals are open available. This fact increases the number of people being able to manufacture receivers, and this will definitely impact the market price of the high precision receivers.

The author thinks, it will be normal to find dual frequency receivers 10 times cheaper than the present ones. Which means receivers between $1000 €$ and $3000 €$.

\section{Conclusions}

In next 5 years the number of positioning systems and satellites will be highly increase.

The huge number of GNSS satellites available, will improve PDOP value worldwide and, more important, will make it more homogenous.

In flight test application, this will alleviate the lost of solution when performing bank turns or stall maneuvers.

Number of available signals will be also increased and all of them will include a nonmodulated pilot signal. In addition, the minimum power received will be increased and the spreading codes will be 10 times faster in some cases, providing a better resolution in pseudodistance measurements to satellites. These factors will make easier to acquire and track positioning signals.

It is important to notice that the L5 and E5 frequency allocation, will protect this signals against interferences, but will complicate the installation of flight test antennas, due to possible interferences with other aircraft systems.

In addition, and to improve GNSS figures against multipath, new improved modulations have been design. The main improvements are more signal bandwidth (but not at the cost of complicating receivers too much) and a steeper correlation peak slope. These multipath improvements will allow a better performance in the high multipath environment of a metallic aircraft.

It is quite reasonable to think that future GNSS receivers used in flight test applications will use GPS and GALILEO as constellations and L1, E1, L5 and E5a as positioning signals.

Finally, due to the new open accessible signals, many people will be able to create GNSS receivers, making the high end market around 10 times cheaper.

\section{References}

[1] N. Linty, Codeless Tracking Algorithms for GNSS Software Receivers, Politecnico Di Torino.

[2] K.Borre et. Al, A Software-Defined GPS and GALILEO Receiver: A Single Frequency approach, Editor: Birkhäuse Boston, 2007. ISBN: 978-0817643904.

[3] European Space Agency, European GNSS Open Service - Signal in Space Interface Document, Issue 1.1, September 2010.

[4] C.Lee et. al, Multipath Benefits of BOC vs. BPSK Modulated Signals using On-Air Measurements, International Technical Meeting of ION, 2013.

\section{Appendix.- MBOC modulation construction}

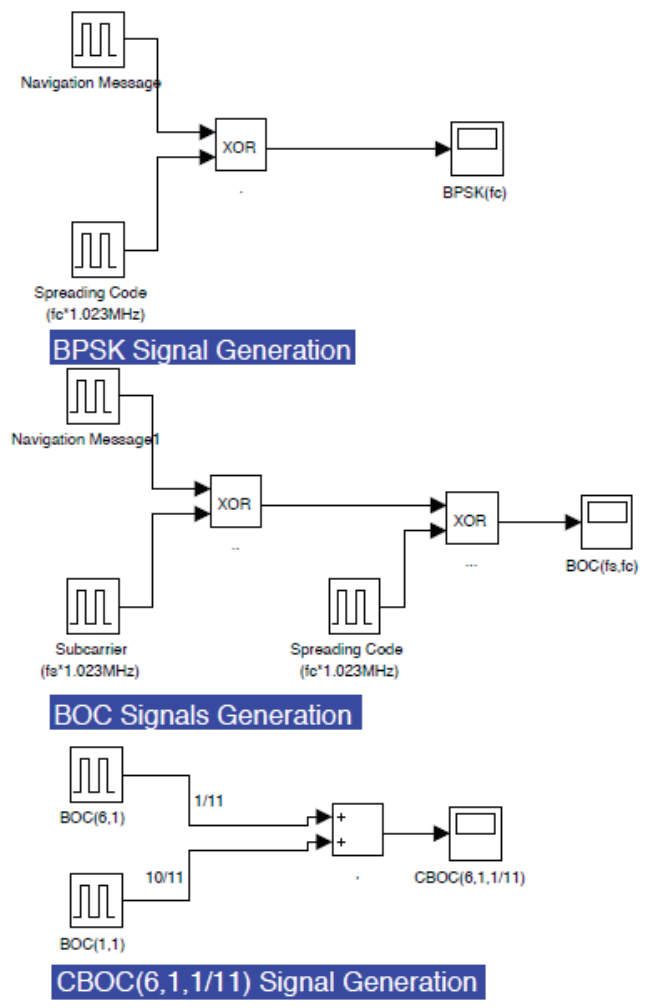

Fig. 11.- BOC Modulations generation

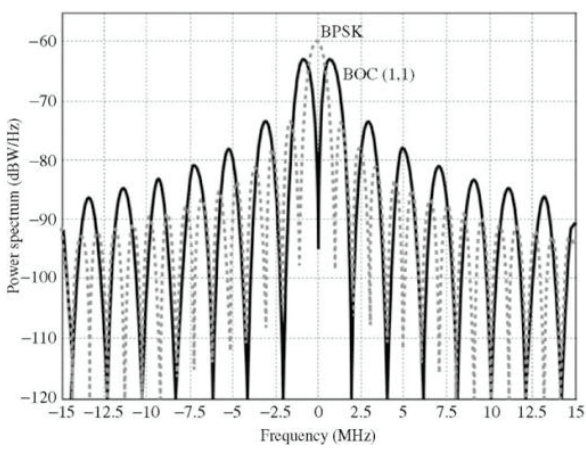

Fig. 12.- $B O C(1,1)$ vs BPSK(1) Spectrum 